\title{
Syntaxonomical Synopsis of the Steppe Vegetation of Turkey
}

\author{
Türkiye'nin Step Vejetasyonunun Sintaksonomik Özeti
}

\author{
Ebru Özdeniz \\ Department of Biology, Faculty of Science, Ankara University, Ankara, Turkey
}

\begin{abstract}
Floristic, ecological and genetic diversity is extremely important in terms of the Anatolian steppe (Turkey) that is located in the western border of Irano-Turanian floristic region. Syntaxonomy of Anatolian steppe have been studied over 40 years but there were many invalid published names and a disorder among syntaxonomical units. In this paper it was aimed to overview the phytosociological studies and the sum of syntaxa of steppe vegetation of Turkey. In the study, all syntaxa described so far from steppe vegetation of Turkey were gathered together to clear their ranks and last status. Steppe vegetation in Turkey is evaluated in 7 class, 13 order, 6 suborder, 52 alliance and 5 suballiance.
\end{abstract}

Key words: steppe vegetation, syntaxonomy, Turkey.

Özet: Iran-Turan floristik bölgesinin batı sınırında yer alan Anadolu stebi (Türkiye), floristik, ekolojik ve genetik çeşitlilik açısından son derece önemlidir. Anadolu stebinin sintaksonomisi 40 yıldır çalışılmaktadır ancak sintaksonomik birimler arasında bir düzensizlik ve birçok geçersiz yayınlanmış isim bulunmaktadır. Bu makalede, Türkiye'nin step vejetasyonuna ait sintaksonlar özetlenmeye ve bu konudaki fitososyolojik çalışmaların kısaca gözden geçirilmesi amaçlanmıştır. Bu çalışmada bugüne kadar tanımlanan Türkiye'nin step vejetasyonuna ait bütün sintaksonlar kademeleri ve son statülerini saptamak üzere biraraya toplanmıştır. Türkiye'deki step vejetasyonu 7 sinif, 13 ordo, 6 alt ordo, 52 alyans ve 5 alt alyans ile temsil edilmektedir.

Anahtar sözcükler: step vejetasyonu, sintaksonomi, Türkiye.

\section{Introduction}

Steppe is a xeric plant formation drying out in summer and dormant in winter, dominated by the cushion forming chamephytes, annual and perennial grasses. The steppe vegetation covering the vast areas in Central Asia extends towards to Inner Anatolia in the west.

Anatolian steppes differs from the Asian steppes by their anthoropogenic origin due to the destruction of pine (Pinus) and oak (Quercus) forests in Anatolia (Akman, 1974). While the grasses (Gramineae) are dominated in Asian steppes where a continental climate prevails, broad and fleshy leaved (malacophyllous) plants are dominant in Anatolia, under the effects of Mediterranean climate (Takhtajan, 1986).

The steppe vegetation shows an altitudinal variation. Therefore, those occupying flat and slightly sloped lands between 800-1200 meters are called as 'plain steppe' and above this elevations those spreading on the steeper slopes are called 'montane steppes'.

On the other hand, steppe formations in Turkey can be groupped into four clusters according to their aspects;

i. Malacophyllous steppes: It is beleived to be derived from grass steppe dominated by fleshy leaved plants as a result by overgrazing on more humid and deeper soils.

ii. Gramineae steppes: This type of steppe dominated by grasses are no longer common today.

iii. Tragaganthic steppes: These are the steppes mostly dominaned by cushion forming spiny species such as Astragalus and Acantholimon. 
iv. Salty steppes: In addition to those above, this is a type formed by halophytic plants occupying saline areas. The widespread species here belong to the families Amaranthaceae (syn: Chenopodiaceae) and Plumbaginaceae (Kurt et al, 2006).

Steppe vegetation in Turkey occupies large areas of Central, Eastern and South-Eastern Anatolia. These regions are the utmost western extension of Irano-Turanian floristic region phytosociologically.

Except for the Irano-Turanian parts of Turkey, highland steppes are dominated above the timberline of Taurus in the South and North Anatolian mountain range.

The first attempts for classification of syntaxonomic units of steppe vegetation in Turkey date back to 1960s. Many studies of Birand, Çetik, Akman, Quézel, Ketenoğlu, Kurt are focused on the steppe vegetation in Anatolia. After the 1990s, syntaxonomical studies on steppe vegetation of Anatolia has accelerated and many syntaxa were published. However, this sitution has created a confusion in terms of compliance with the international code of phytosociological nomenclature.

In this article, the syntaxa described so far are complied and the upper units are rearranged.

\section{Results and Discussion}

Phytosociological steppe units of Turkey following list with class, order, alliance and associations was prepared and gathered together to clear their ranks and last status. Syntaxonomic units of stepe vegetation of Turkey is evaluated in 7 class, 13 order, 6 suborder, 52 alliance and 5 suballiance. Class, order and alliance are illustrated schematically.

\section{Class: DAPHNO OLEOIDIS-FESTUCETEA VARIAE Quézel 1964}

This class occupies high mountainous areas in the north and north-western Anatolia.

Characteristic species; Festuca cyllenica Boiss. \& Heldr. [Syn: Festuca varia subsp. cyllenica (Boiss. \& Heldr.) Acht.], Festuca valesiaca Schleich. ex Gaudin [Syn: Festuca ovina L.], Festuca rubra L., [Syn: Festuca ovina var. duruiscula (L.) W.D.J.Koch], Rostraria cristata (L.) Tzelev [Syn: Koeleria cristata (L.) Bertol.], Stipa pulcherrima K. Koch., Daphne oleoides Schreb., Aubrieta deltoidea L. (DC.), Iberis sempervirens L., Berberis cretica L., Paronychia chionaea Boiss., Telephium imperati subsp. orientale (Boiss.) Nyman. [Syn: Telephium orientale Boiss.], Prunus prostrata Labill., Astragalus angustifolius Lam., Acantholimon ulicinum (Willd. ex Schult.) Boiss. [Syn: Acantholimon echinus (L.) Boiss., Acantholimon androsaceum (Jaub. \& Spach) Boiss.], Cruciata taurica subsp. persica (DC.) Ehrend., Scutellaria orientalis L., Asyneuma limonifolium (L.) Janch., Thymus roegneri K. Koch. [Syn: Thymus hirsutus M. Bieb.]

1.0.1. Alliance: Hyperico-Verbascion Akman \& Ketenoğlu 1978

Locality; Köroğlu Mountain, Kös Mountain, Halkoluk Plateau/Between Devrez Stream and Kızılırmak River/Transition zone of Western Black Sea Region and Central Anatolia (Kılınç, 1985)

Bedrock; Andesite

Altitude; $1500-2350 \mathrm{~m}$

Characteristic \& Diagnostic species; Verbascum armenum Boiss. \& Kotschy subsp. occidentale Hub-Mor., Astragalus amoenus subsp. squalidus (Boiss.) Ponert [Syn: Astragalus squalidus Boiss.], Jasione supina Sieber ex Spreng. subsp. akmanii Dambolt, Hypericum orientale L., Sideritis germanicopolitana Bornm., Cerastium purpurascens Adams, Genista tinctoria L., Hypericum linarioides Bosse, Marrubium astracanicum Jacq., Stachys iberica M. Bieb., Stachys germanica subsp. bithynica (Boiss.) R. Bhattacharjee, Astragalus aquilonius (Barneby) Barneby, Pilosella pilisquama (Nägeli \& Peter) Dostál [Syn: Pilosella hopeana subsp. troica (Zahn) P.D.Sell \& C.West]

\subsection{Order: DAPHNO OLEOIDIS-FESTUCETALIA VARIAE Quézel 1964}

This order that is located in Greece and in the west of Anatolia characterizes mesogeenes floristically and Mediterranean mountains climatically (Barbero et al. 1975). It spreads Akdağ/AfyonDenizli environs Ayaş Mountains and Bozdağ/İzmir (Akman and Ketenoğlu 1976; Gemici, 1988; Bekat and Oflas, 1990). 
Characteristic \& Diagnostic species; Acantholimon puberulum Boiss. \& Balansa subsp. puberulum, Helichrysum pallasii (Spreng.) Ledeb., Marrubium astracanicum Jacq., Euphorbia herniariifolia Willd. subsp. herniariifolia, Minuartia globulosa (Labill.) Schinz \& Thell., Eremogone ledebouriana (Fenzl) Ikonn. [Syn: Arenaria ledebouriana Fenzl.], Saponaria pumilio Boiss., Eryngium bourgatii subsp. heldreichii (Boiss.) P.H. Davis, Dianthus erinaceus Boiss. var. alpinus, Juniperus communis var. saxatilis Pall. [Syn: Juniperus communis subsp. nana (Willd.) Baumg.], Sideritis condensata Boiss. \& Heldr., Draba brunifolia Steven, Silene caryophylloides Otth, Erysimum kotschyanum J. Gay [Syn: Erysimum pallidum Boiss.], Muscari bourgaei Baker, Merendera trigyna Woronow, Ranunculus demissus DC. var. major Boiss.

1.1.1. Alliance: Stipo pulcherrimae-Morinion persicae Quézel 1964

Locality; Gavur Mountains/Erzurum (Tat11 1985)

Bedrock; Calcareous

Altitude; 2250-2360 m

Characteristic \& Diagnostic species; Stipa pulcherrima K.Koch [Syn: Stipa pennata var. pulcherrima (K. Koch) Asch. \& Graebn.], Scutellaria altissima L. [Syn: Scutellaria peregrina Waldst.\&Kit.], Melica ciliata L., Pterocephalus perennis Coult., Asphodeline lutea (L.) Reichb. [Syn: Asphodelus luteus L.], Anthemis cretica L. [Syn: Anthemis montana L.], Ononis pusilla L., Echinops sphaerocephalus subsp. taygeteus (Boiss. \& Heldr.) Kožuharov, Morina persica $L$.

1.1.2. Alliance: Festucion chalcophaeae Tatl 1982

Locality; Dumlu and Gavur Mountains/Erzurum, Tahir Mountain and Güzeldere Valley/Ağr1 (Behçet \& Tatlı 1989; Gümüş 1992)

Bedrock; Andesite, basalt, calcareous, schist, marn

Altitude; 2000-2250 m

Characteristic \& Diagnostic species; Festuca chalcophaea Krecz. \& Bobrov, Draba bruniifolia Steven, Tripleurospermum oreades (Boiss.) Rech. f. var. oreades, Minuartia recurva (All.) Schinz \& Thell. subsp. oreina (Mattf.) McNeill, Erigeron caucasicus Steven, Plantago atrata Hoppe

2. Class: ASTRAGALO-BROMETEA Quézel 1973

This class containing thorny cushion-like chamaephytes and perennial Gramineae plants extends across the Taurus mountains and the Aegean Anatolian plateau. It envelops all mountainous areas of Anatolia. The characteristic species of the class belong to are essentially oro-Mediterranean zone (Akman et al, 1987). It is represented in Turkey by six orders.

Characteristic species; Bromus tomentellus Boiss., Anthyllis variegata Grossh. [Syn: Anthyllis vulneraria subsp. variegata (Boiss.) Cullen], Rostraria cristata (L.) Tzelev [Syn: Koeleria cristata (L.) Bertol.], Daphne oleoides Schreb., Leontodon asperrimus (Willd.) Endl., Scutellaria orientalis L., Draba bruniifolia Steven, Asyneuma virgatum (Labill.) Bornm., Senecio pseudo-orientalis Schischk., Helianthemum nummularium (L.) Mill., Androsace villosa L., Poa alpina L. [Syn: Poa alpina subsp. fallax F.Hermann], Helichrysum plicatum DC., Globularia trichosantha Fisch. \& C.A.Mey., Astragalus (plural sp.), Campanula stricta L., Veronica multifida L., Sideritis libanotica Labill., Cyanus triumfettii subsp. axillaris (Čelak.) Štěpánek [Syn: Centaurea triumfettii var. cana (Sibth. \& Sm.) Dostál], Podospermum canum C.A.Mey. [Syn: Scorzonera jacquiniana (W.D.J.Koch) Boiss.], Linum tenuifolium L., Acantholimon ulicinum (Willd. ex Schult.) Boiss. [Syn: Acantholimon echinus (L.) Boiss., Acantholimon androsaceum (Jaub. \& Spach) Boiss.], Ononis adenotricha Boiss., Helianthemum oelandicum subsp. incanum (Willk.) G.López [Syn: Helianthemum canum (L.) Baumg.], Teucrium chamaedrys L., Minuartia anatolica (Boiss.) Woronow, Hypericum origanifolium Willd., Cruciata taurica subsp.taurica [Syn: Cruciata coronata (Sm.) Ehrend.], Ziziphora clinopodioides Lam., Papaver apokrinomenon Fedde, Teucrium polium L., Iberis simplex DC. [Syn: Iberis taurica DC.], Melica ciliata L., Onobrychis cornuta (L.) Desv., Phlomis armeniaca Willd., Silene spergulifolia (Willd.) M.Bieb., Minuartia juniperina (L.) Maire \& Petitm., Astragalus angustifolius Lam., Euphorbia myrsinites L., Anthemis cretica L. [Syn: Anthemis montana L.] 
The two alliances below (Astragalo erythrotaeni-Gundelion armatae and Tanaceto orientaliAstragalion gummiferi) are attached directly to the class Astragalo-Brometea.

2.0.1. Alliance: Astragalo erythrotaeni-Gundelion armatae Kaya \& Ketenoğlu 2010

Locality; Karacadağ Mountain/Şanlıurfa-Diyarbakır (Kaya \& Ketenoğlu 2010)

Bedrock; Volcanic, Basalt

Altitude; $1000-1500 \mathrm{~m}$

Characteristic \& Diagnostic species; Alcea striata (DC.) Alef subsp. striata, Astragalus amblolepis, Astragalus erythrotaenius Boiss., Astragalus plumosus subsp. akardaghicus (Eig) Ponert [Syn: Astragalus plumosus Willd. var. akardaghicus (Eig) D.F. Chamb. \& Matthews], Cerasus microcarpa (C.A. Mey.) Boiss. subsp. tortuosa (Boiss. \& Hausskn.) Browicz, Cicer echinospermum P.H. Davis, Crucianella exasperata Fisch. \& C.A. Mey., Dianthus hymenolepis Boiss., Dianthus strictus Banks \& Sol. var. gracilior (Boiss.) Reeve, Grammosciadium macrodon Boiss., Gundelia tournefortii L. var. armata Freyn \& Sint., Lathyrus inconspicuus L. var. stenophyllus (Boiss.) Rech., Lens montbretii (Fisch. \& C.A. Mey.) P.H. Davis \& Plitmann, Lotus gebelia Vent. var. hirsutissimus (Ledeb.) Dinsm., Medicago shepardi Post ex Boiss., Onobrychis megataphros Boiss., Onosmo rascheyanum Boiss., Paracaryum cristatum (Schreb.) Boiss. subsp. cristatum, Scrophularia libanotica Boiss. subsp. armena R.R. Mill, Torilis leptocarpa (Hochst.) C.C. Towns., Trifolium hausknechtii Boiss. var. candollei (Post) Hossain.

2.0.2. Alliance: Tanaceto orientali-Astragalion gummiferi Kaya \& Ketenoğlu 2010

Locality; Karacadağ Mountain/Şanlıurfa-Diyarbakır (Kaya \& Ketenoğlu 2010)

Bedrock; Volcanic, Basalt

Altitude; 1500-1950 m

Characteristic \& Diagnostic species; Alcea calvertii (Boiss.) Boiss., Astragalus gummifer Labill., Astragalus lagopoides Lam. [Syn: Astragalus lagurus Willd.], Delphinium macrostachyum Boiss. ex Huht, Echinops heterophyllus P.H. Davis, Fibigia macrocarpa (Boiss.) Boiss., Linaria kurdica Boiss. \& Hohen. subsp. araratica (Tzvelev) P.H. Davis, Paracaryum kurdistanicum (Brand) R.R. Mill, Phlomis armeniaca Willd., Phlomis kurdica Rech.f., Ranunculus macrorhynchus Boiss. subsp. trigonocarpus (Boiss.) P.H. Davis, Rosa vanheurckiana Crép. var. vanheurckiana [Syn: Rosa heckeliana Tratt. subsp. vanheurckiana (Crép.) O. Nilsson], Symphytum aintabicum Hub.- \& Wickens, Tanacetum cadmeum (Boiss.) Heywood subsp. orientale Grierson, Telephium oligospermum Steud. ex Boiss., Thymus kotschyanus Boiss. \& Hohen. [Syn: Thymus kotschyanus Boiss. \& Hohen. var. glabrescens Boiss.], Viola modesta Fenzl. Mor.

2.1. Order: ASTRAGALO-BROMETALIA Quézel 1973

The order extends in the central Taurus, western part of eastern Taurus range, and a mountain range in northwest Anatolia (Quézel, 1973; Akman et al, 1983; Ocakverdi and Çetik, 1982).

Characteristic \& Diagnostic species; Bromus tomentellus Boiss., Onobrychis cornuta (L.) Desv., Rumex angustifolius Campd. subsp. macranthus (Boiss.) Rech.f., Ononis adenotricha Boiss., Polygonum setosum Jacq., Cynoglossum montanum L., Aethionema iberideum (Boiss.) Boiss., Paracaryum lithospermifolium (Lam.) Grande, Silene cappadocica Boiss. \& Heldr., Campanula stricta L., Silene sperguliflolia (Willd.) M.Bieb., Scorzonera cana (C.A. Mey.) Griseb. var. jacquiniana (W. Koch) D.f. Chamb., Minuartia juniperina (L.) Maire \& Petitm., Phlomis armeniaca Willd., Minuartia anatolica (Boiss.) Woronow, Senecio pseudo-orientalis Schischk., Astragalus species.

2.1.1. Alliance: Tanacion praeteriti Quézel 1973

Locality; Akdağ, Tekedağ, Sandras Mountain, West of Antalya/Western Taurus (Quézel, 1973)

Bedrock; Serpentine, Calcareous

Altitude; $1700-2700 \mathrm{~m}$

Characteristic \& Diagnostic species; Dianthus eretmopetalus Stapf., Astragalus paecilanthus Boiss. \& Heldr., Bornmuellerantha aucheri (Boiss.) Rothm. [Syn: Odontites aucheri Boiss.], Tanacetum praeteritum (Horw.) Heywood, Anthemis rosaea Sm., Elymus reflexiarristatus (Nevski) Melderis [Syn: Agropyron strigosum (Schult.) Boiss.] 
2.1.2. Alliance: Thurion capitatae Quézel 1973

Locality; Mazmılı Mountain, Acıman Plateau (Quézel, 1973)

Bedrock; Calcareous

Altitude; 2000-2500 m

Characteristic \& Diagnostic species; Alyssum callichroum Boiss., Cochlearia sempervivum Boiss. \& Balansa, Thlaspi rosulare Boiss. \& Balansa, Thurya capitata Boiss. \& Balansa, Fumana oligosperma Boiss. \& Kotschy, Sideritis phlomoides Boiss. \& Balansa, Scutellaria diffusa Benth., Leontodon asperrimus (Willd.) Endl. [Syn: Leontodon masmenaeus Boiss. \& Balansa], Lactuca mulgedioides (Vis. \& Pančić) Boiss. \& Kotschy ex Boiss. \& Kotschy [Syn: Lactuca seticuspis Boiss.]

2.1.3. Alliance: Paronychion lycicae Quézel 1973

Locality; Akdağ/Western Taurus (Quézel, 1973)

Bedrock; Calcareous

Altitude; 2200-2600 m

Characteristic \& Diagnostic species; Alopecurus lanatus Sm., Paronychia lycica Chaudhri, Dianthus brevicaulis Fenzl. subsp. setaceus Reeve, Veronica cuneifolia D. Don, Asperula lycia Stapf.

2.1.4. Alliance: Silenion oreades Quézel 1973

Locality; Akseki, Yaylacık Hill, Crrlavık Hill, Ermenek and Oyuklu Mountain/Central Taurus, Bolkar mountains (Quézel, 1973)

Bedrock; Calcareous

Altitude; 2200-2600 m

Characteristic \& Diagnostic species; Fritillaria crassifolia Boiss. \& A.Huet, Aethionema subulatum (Boiss. \& Heldr.) Boiss., Erysimum kotschyanum J.Gay, Arenaria isaurica Boiss., Silene oreades Boiss. \& Heldr., Veronica cuneifolia D.Don subsp. isaurica P.H. Davis, Achillea formosa (Boiss.) Sch. Bip. [Syn: Leucocyclus formosus Boiss.]

2.2. Order: DRABO-ANDROSACETALIA Quézel 1973

This order extends on calcareous bedrock of alpine and subalpine zones of Taurus Mountains, Davras Mountain/Isparta, Anti-Taurus Mountains /Adana (Sağlam 2007; Yurdakuol 1973). It is hard to draw the farthest boundaries of the order, but there is a possibility of presence of the order in high elevations of Lebanon Mountains (Akman et al, 2014).

Bedrock; Calcareous

Altitude; 2100-2300 m

Characteristic \& Diagnostic species; Alopecurus vaginatus (Willd.) Kunth, Aethionema cordatum (Desf.) Boiss., Festuca pinifolia (Hack. ex Boiss.) Bornm., Astragalus angustifolius Lam. subsp. pungens (Willd.) Hayek, Minuartia leucocephala Mattf., Saponaria pumilio (L.) Fenzl ex A.Braun, Alyssum pateri Nyár., Androsace villosa L., Alyssum oxycarpum Boiss. \& Balansa, Pedicularis cadmea Boiss., Alyssum lepidotum Boiss., Veronica caespitosa Boiss. var. caespitosa, Alyssum condensatum Boiss. \& Hausskn., Ziziphora clinopodioides Lam., Alyssum condensatum subsp. flexibile (Nyár.) Dudley, Asperula setosa Jaub. \& Spach, Alyssum erosulum Gennari \& Pestal. ex Clem., Psephellus mucronifer (DC.) Wagenitz [Syn: Centaurea mucronifera DC.], Hesperis kotschyi Boiss., Centaurea kotschyi (Boiss. \& Heldr.) Hayek, Draba brunifolia subsp. heterocoma (Fenzl) Coode \& Cullen, Centaurea drabifolia subsp. cappadocica (DC.) Wagenitz, Draba brunifolia Steven subsp. brunifolia, Centaurea drabifolia Sibth.\&Sm. subsp. austro-occidentalis Wagenitz, Aethionema oppositifolium (Pers.) Hedge

2.1.1. Alliance: Agropyro-Stachion Quézel 1973

Locality; Akseki, Ermenek, Oyuklu Mountain, Bolkar Mountains/West of Antalya

Bedrock; Calcareous

Altitude; $1700-2300 \mathrm{~m}$

Characteristic \& Diagnostic species; Elymus tauri (Boiss. \& Bal.) Melderis [Syn: Agropyron tauri Boiss. \& Balansa], Hypericum scabrum L., Asphodeline taurica (Pall.) Endl., Onosma stellulatum 
Waldst. \& Kit., Papaver polychaetum Schott \& Kotschy ex Boiss., Pterocephalus pinardi Boiss., Arenaria rotundifolia M.Bieb. [Syn: Arenaria pauciflora (Boiss.) Prodán], Galium cilicicum Boiss., Eremogone acerosa (Boiss.) Ikonn. [Syn: Arenaria acerosa Boiss. \& Heldr.], Asyneuma lobelioides (Willd.) Hand.-Mazz., Silene spergulifolia (Willd.) M.Bieb. [Syn: Silene supina M.Bieb.], Asyneuma virgatum (Labill.) Bornm., Aethionema coridifolium DC., Senecio pseudoorientalis Schischk., Hypericum kotschyanum Boiss., Stachys lavandulifolia Vahl

2.3. Order: ONOBRYCHIDO ARMENI-THYMETALIA LEUCOSTOMI Akman, Ketenoğlu, Quézel 1985

This order includes all steppe associations developed under the xeric climatical conditions are in the central and peripheral zone of Anatolia. The altitudinal ranges of the order vary between $600-1300$ meters. The floristic composition of steppe vegetation of Central Anatolia does not match up with the floristic composition of Taurus and Anti-Taurus Mountains. Main reasons of the variation are height difference, precipitation, bedrock and endemism rate.

Characteristic \& Diagnostic species; Onobrychis arenaria subsp. cana (Boiss.) Hayek [Syn: Onobrychis armena Boiss. \& A.Huet], Alyssum pateri Nyár., Minuartia hamata (Hausskn.) Mattf., Stachys cretica L., Alyssum sibiricum Willd., Marrubium parviflorum Fisch. \& C.A.Mey., Moltkia caerulea Lehm., Paronychia kurdica Boiss., Dianthus anatolicus Boiss., Polygala anatolica Boiss. \& Heldr., Polygala pruinosa Boiss., Globularia orientalis L., Anthemis tinctoria L., Centaurea virgata Lam., Astragalus condensatus Ledeb., Achillea santolinoides subsp. wilhelmsii (K.Koch) Greuter [Syn: Achillea wilhelmsii K.Koch], Achillea phrygia Boiss. \& Balansa, Ajuga salicifolia (L.) Schreb., Astragalus lycius Boiss., Astragalus mesogitanus Boiss. [Syn: Astragalus lydius Boiss.], Bungea trifida (Vahl) C.A.Mey., Convolvulus holosericeus M. Bieb., Cousinia birandiana Hub.-Mor., Genista aucheri Boiss., Genista sessilifolia DC., Hedysarum varium Willd., Hypericum aviculariifolium Jaub. \& Spach, Jurinea consanguinea DC., Lappula barbata (M.Bieb.) Gürke, Linum flavum L., Noaea mucronata (Forssk.) Asch. \& Schweinf., Onobrychis hypargyrea Boiss., Onosma aucherana DC., Onosma isaurica Boiss. \& Heldr., Phlomis pungens Willd., Salvia cryptantha Montbret \& Aucher ex Benth., Salvia hypargeia Fisch. \& C.A.Mey., Bromus cappadocicus Boiss. \& Balansa subsp. cappadocicus, Silene supina M.Bieb. subsp. pruinosa (Boiss.) Chowdhuri, Astragalus brachypterus Fisch., Fumana aciphylla Boiss., Thymus leucostomus Hausskn. \& Velen., Saponaria prostrata Willd., Astragalus anthylloides Lam., A. campylosema Boiss. , A. vulnerariae DC., Leontodon asperrimus (Willd.) Endl.

2.3.0.1. Alliance: Cousinia iconici-Artemision santonici Geven, Adıüzel and Vural 2010

Locality; Ereğli-Karaman (Geven et al, 2010)

Bedrock; Calcareous

Altitude; $1000-1180 \mathrm{~m}$

Characteristic \& Diagnostic species; Artemisia santonicum L., Cousinia iconica Hub.-Mor., Androsace maxima L., Loliolum subulatum (Banks \& Sol.) Eig, Alyssum linifolium Stephan ex Willd., Consolida stenocarpa (P.H.Davis \& M.Hossain) P.H.Davis, Minuartia sclerantha Thell., Allium macrochaetum Boiss. \& Hausskn., Ceratocephalus falcatus (L.) Pers.

2.3.0.2. Alliance: Euphorbio armeni-Oligochaetion divaricatae Tatll 1991

Locality; Karahacılı, Gödekli villages/Iğdır/East Anatolia (Tatlı, 1991)

Bedrock; Volcanic

Altitude; 800-1000 m

Characteristic \& Diagnostic species; Oligochaeta divaricata (Fisch. \& C.A.Mey.) K.Koch, Stipa hohenackeriana Trin. \& Rupr., Euphorbia marschalliana subsp. armena (Prokh.) Oudejans [Syn: Euphorbia armena Prokh.], Trinia scabra Boiss. \& Noë, Erysimum huber-morathii Polatschek [Syn: Erysimum torulosum Hub.-Mor.], Stipagrostis plumosa Munro ex T.Anderson, Lepidium vesicarium L.

2.3.1. Suborder: ONOBRYCHIDO ARMENIAE-THYMENETALIA LEUCOSTOMI Akman, Quézel, Barbéro, Ketenoğlu and Aydoğdu 1991

This suborder extends on lower elevations (1200-1300 m) of central and south-west Anatolia. 
Characteristic \& Diagnostic species; Artemisia scoparia Waldst. \& Kitam., Cousinia birandiana Hub.-Mor., Thymus leucostomus Hausskn. \& Velen., Artemisia santonicum L., Bungea trifida (Vahl) C.A.Mey., Astragalus mesogitanus Boiss. [Syn: Astragalus lydius Boiss.], Stipa holosericea Trin., Salvia absconditiflora Greuter \& Burdet [Syn: Salvia cryptantha Montbret \& Aucher ex Benth.], Stipa lessingiana Trin. \& Rupr.

1.3.1.1. Alliance: Alysso lepidoto-stellati-Astragalion condensati Aydoğdu, Ketenoğlu and Hamzaoğlu 1999

Locality; Baran and Kervansaray Mountain/Kırşehir (Aydoğdu, 1999)

Bedrock; Calcareous

Altitude; $1380-1670 \mathrm{~m}$

Characteristic \& Diagnostic species; Astragalus condensatus Ledeb., Astragalus micropterus Fischer, Astragalus densifolius Lam., Alyssum lepidoto-stellatum (Hausskn. \& Bornm.) Dudley, Euphorbia anacampseros Boiss., Salvia modesta Boiss., Verbascum vulcanicum Boiss. \& Heldr. var. vulcanicum, Minuartia anatolica (Boiss.) Woron. var. arachnoidae McNeill, Anchonium elichrysifolium (DC.) Boiss. subsp. canescens (Hausskn. ex Bornm) Jacquemoud, Sideritis galatica Bornm., Salvia blepharochlaena Hedge \& Hub.-Mor., Centaurea paphlagonica (Bornm.) Wagenitz.

1.3.1.2. Alliance: Convolvulo assyrici-Helianthemion cani Ketenoğlu, Aydoğdu, Kurt, Hamzaoğlu, Tuğ \&Aslantürk 2008

Locality; Şarkışla, Kangal and Gürün/Sivas (Ketenoğlu et al, 2008)

Bedrock; Calcareous

Altitude; $1300-1700 \mathrm{~m}$

Characteristic \& Diagnostic species; Helianthemum oelandicum subsp. incanum (Willk.) G.López [Syn: Helianthemum canum (L.) Baumg.], Ebenus laguroides Boiss., Hypericum thymopsis Boiss., Convolvulus assyricus Griseb., Convolvulus compactus Boiss., Linum flavum L. subsp. scabrinerve (Davis) Davis, Galium incanum Sm. subsp. pseudocornigerum Ehrend., Stipa capillata L., Minuartia anatolica (Boiss.) Woronow subsp. scleranthoides (Boiss. \& Noë) McNeill, Asphodeline tenuior (Fisch.) Ledeb. subsp. tenuiflora (K.Koch) E.Tuzlac1

1.3.1.3. Alliance: Convolvulo holosericei-Ajugion salicifoliae Akman, Ketenoğlu, Quézel \& Demirörs 1984 1984)

Locality; West of Ankara, Ayaş, Temelli, Polatlı and Beypazarı, Acıkır, Polatlı/Ankara (Akman,

Bedrock; Marly and marly-gypsaceous

Altitude; $650-1150 \mathrm{~m}$

Characteristic \& Diagnostic species; Convolvulus holoseriseus Bieb. subsp. holosericeus, Ajuga salicifolia (L.) Schreber, Euphorbia macroclada Boiss., Linum flavum L. subsp. scabrinerve (Davis) Davis, Galium verum L.

2.3.1.3.1. Suballiance: Ebeno hirsutae-Thymenion leucostomi Hamzaoğlu, Aydoğdu, Kurt and Cansaran 2004

Locality; Cihanbeyli/Konya, Çifteler/Eskişehir (Hamzaoğlu et al, 2004)

Bedrock; Calcareous

Altitude; 850-1150 m

Characteristic \& Diagnostic species; Thymus leucostomus Hausskn \& Velen, Ebenus hirsuta Jaub. \& Spach, Minuartia anatolica (Boiss.) Woron. var. arachnoidea McNeill, Astragalus vulnerariae DC., Cousinia iconica Hub.- Mor., Astragalus tmoleus Boiss. var. bounacanthus (Boiss.) Ponert., Alyssum niveum Dudley, Achillea phrygia Boiss. \& Bal.

1.3.1.4. Alliance: Salvio tchihatcheffii-Hedysarion varii Akman, Ketenoğlu, Quézel \& Demirörs 1984

Locality; South-west of Ankara, Haymana (Akman et al, 1984)

Bedrock; Marly

Altitude; 800-1250 m 
Characteristic \& Diagnostic species; Salvia tchihatcheffi (Fisch. \& Meyr.) Boiss., Hedysarum varium Willd., Linum hirsutum L. subsp. anatolicum (Boiss.) Hayek var. anatolicum, Helianthemum nummularium (L.) Mill., Asyneuma limonifolium (L.) Janch.

2.3.1.5. Alliance Phlomido armeniacae-Astragalion microcephali Akman, Ketenoğlu, Quézel \& Demirörs 1984

Locality; South-west of Ankara, Beynam-Bala, Ayaş Mountains west of Ankara, Kırıkkale, Kalecik, Kirşehir, Davras Mountain, Çiçek Mountain, Kızıldağ/Isparta, Karadağ/Karaman, Ermenek, Tahir Mountain and Güzeldere Valley/Erzurum, Emirdağ/Afyon, Beykavağ1 Kestel Mountain/Burdur, Kızılören-Çal-Loras, Akdağ, Ilgın, Kadınhanı, Hadim, Ermenek, Bucakkışla/Konya, Kütahya, Karaömer Mountain/Amasya, Pirreşit Mountain/Muradiye-Van, Baskil/Elazı ̆̆ (Akman and Ketenoğlu1976; Akman et al, 1996; Gümüş, 1992; Tatlı et al, 1994; Akman et al, 1996; Behçet and Ünal, 1999; Kurt, 2002; Sağlam, 2004; Sağlam, 2007).

Bedrock; Radiolarite, flysch, marly, serpantine

Altitude; 750-1300 m

Characteristic \& Diagnostic species; Phlomis armeniaca Willd., Astragalus microcephalus subsp. microcephalus, Teucrium chamaedrys L., Marrubium parviflorum Fisch. \& Meyr. subsp. oligodon Boiss.

2.3.1.5.1. Suballiance: Astragalenion lycii Akman, Ketenoğlu, Quézel \& Demirörs 1984

Locality; Karayavşan, Şerefliköy, Yenice, Haymana/Ankara (Akman et al, 1984)

Bedrock; Flysch

Altitude; $1000 \mathrm{~m}$

Characteristic \& Diagnostic species; Astragalus lycius Boiss., Helianthemum oelandicum subsp. incanum (Willk.) G.López [Syn: Helianthemum canum (L.) Baumg.], Astragalus lycius Boiss., Acantholimon acerosum (Willd.) Boiss. subsp. acerosum

2.3.1.6. Alliance: Phlomido nissolii-Onobrychdion tournefortii Kurt 2002

Locality; South-west of Afyon, Emirdağ, Bolvadin, Çay, Senirkent, Uluborlu (Kurt, 2002)

Bedrock; Volcanic (agglomerates), chalk, marly chalks

Altitude; 900-1000 m

Characteristic \& Diagnostic species; Hypericum origanifolium var. depilatum (Freyn \& Bornm.) N.Robson [Syn: Hypericum avicularifolium Jaub. \& Spach. subsp. depilatum Freyn \& Bornm.], Phlomis nissolii L., Onobrychis tournefortii (Wild) Desv., Eryngium bithynicum Boiss.

2.3.1.7. Alliance: Astragalo karamasici-Gypsophylion eriocalycis Ketenoğlu, Quézel, Akman \& Aydoğdu 1984

Locality; Polatl1-Acıkır, Beypazarı, Nallıhan, Haymana, north-west of Ankara, Çankırı, Kızılırmak Valley, Yapraklı Mountains/north-east of Çankırı, Şereflikoçhisar-Aksaray,-Nevşehir, Kırıkkkale, Kalecik, Kırşehir (Akman, 1990b; Ketenoğlu et al, 1984)

Bedrock; Gypsaceous, calcareous

Altitude; 350-900 m

Characteristic \& Diagnostic species; Astragalus karamasicus Boiss. \& Ball., Thymus leucostomus Jalas, Linum mucronatum Bertol subsp. gypsicola Davis var. gypsicola, Centaurea patula DC., Allium flavum L. subsp. tauricum (Besser ex Reichb.) Stearn var. tauricum, Gypsophila eriocalyx Boiss., Gypsophila parva Brak, Bupleurum boissieri Post.

2.3.1.7.1. Suballiance: Asperulenion bornmuelleri Ketenoğlu, Quézel, Akman \& Aydoğdu 1983

Locality; North-east part of the Central Anatolia (Ketenoğlu et al, 1983)

Bedrock; Gypcaceous

Altitude; 700-900 m

Characteristic \& Diagnostic species; Asperula bornmuelleri Velen, Acantholimon acerosum (Willd.) Boiss., Minuartia micrantha Schischk., Linaria corifolia Desf., Scorzonera cana var. radicosa (C.A. Meyer) Hoffm. 
2.3.1.7.2. Suballiance: Helichryso-Thymenion cappadoci Ketenoğlu, Aydoğdu, Kurt, Akman \& Hamzaoğlu 2000

Locality; North-east part of the Central Anatolia (Ketenoğlu et al, 2000)

Bedrock; Gypcaceous

Altitude; 700-900 m

Characteristic \& Diagnostic species; Thymus cappadocicus Boiss., Helichrysum noeanum Boiss., Achillea sintenisii Hub.-Mor., Chrysothesium stelleroides (Jaub. \& Spach) Hendrych [Syn: Thesium stellerioides Jaub. \& Spach.], Scorzonera aucheriana DC., Chrysochamela neona (Boiss.) Boiss., Allium sivasicum Özhatay \& Kollman.

2.3.1.8. Alliance: Achilleo wilhelmsii-Artemision santonici Aydoğdu, Kurt, Hamzaoğlu, Ketenoğlu \& Cansaran 2004

Locality; Tuz Lake and Seyfe Lake (Aydoğdu et al, 2004)

Bedrock; Salty soils, salty steppes

Altitude; 960-1000 m

Characteristic \& Diagnostic species; Artemisia santonicum L., Achillea santolinoides subsp. wilhelmsii, (K.Koch) Greuter [Syn: Achillea wilhelmsii K.Koch], Allium pseudoflavum Vved., Reaumuria alternifolia (Lab.) Britten, Alyssum blepharocarpum Dudley and Hub.-Mor., Allium scabriflorum Boiss., Acantholimon halophilum Bokhari, Anthemis fumariifolia Boiss., Verbascum helianthemoides Hub.-Mor.

2.3.1.8.1. Suballiance: Artemisenion santonicii Ketenoğlu, Quézel, Akman \& Aydoğdu 1983

Locality; North-east part of the Central Anatolia (Ketenoğlu et al, 1983)

Bedrock; Gypcaceous, calcareous

Altitude; $7600-800 \mathrm{~m}$

Characteristic \& Diagnostic species; Artemisia santonicum L., Scabiosa argentea L., Taeniatherum caput-medusae (L.) Nevski

2.3.1.9. Alliance: Arenario ledebouriani-Astragalion plumosi Akman 1990

Locality; Aydos Mountain/Ankara, Eldivan Mountain/Çankırı, Dinek Mountain/Kırıkkale (Akman, 1990a; Kurt et al, 1999; Hamzaoğlu and Duran, 2004)

Bedrock; Volkanic, calcareous, marly

Altitude; $1300-1800 \mathrm{~m}$

Characteristic \& Diagnostic species; Eremogone ledebouriana (Fenzl) Ikonn. [Syn: Arenaria ledebouriana Fenzl.], Thymus sipyleus Boiss., Astragalus plumosus Willd., Thymus longicaulis subsp. chaubardii (Rchb.f.) Jalas [Syn: Thymus longicaulis subsp. subisophyllus (Borbás) Jalas], Stachys iberica subsp. stenostachya (Boiss.) Rech. f., Galium verum L. subsp. glabrescens Ehrend., Sideritis germanicopolitana Bornm.

2.3.1.10. Alliance: Minuartion juniperino-pestalozzae Ketenoğlu, Serin, Kurt \& Akman 1996 Locality; Hacıbaba Mountain/Konya (Serin et al, 1996)

Bedrock; Calcareous

Altitude; 2000-2350 m

Characteristic \& Diagnostic species; Minuartia juniperrina (L.) Marie \& Petitm., Minuartia pestallozae (Boiss.) Bornm., Astragalus angustifolius Lam, Marrubium globosum Montbret \& Aucher ex Bentham subsp. micranthum (Boiss. \& Heldr.) P. H. Davis, Silene pharnaceifolia Fenz., Dianthus zederbaueri Vierh., Poa alpina L. [Syn: Poa alpina subsp. fallax F.Hermann], Psephellus mucronifer (DC.) Wagenitz [Syn: Centaurea mucronifera DC.], Veronica cuneifolia D. Don subsp. isaurica P.H. Davis, Paronychia davisii Chaudhri.

2.3.1.11. Alliance: Thymo subisophyllii-Alyssion virgatii Akman, Quézel, Aydoğdu, Ketenoğlu, Kurt \& Evren 1994

Locality; Yapraklı Mountain/Çankırı (Akman et al, 1994)

Bedrock; Calcareous, serpentine

Altitude; $1400-1850 \mathrm{~m}$ 
Characteristic \& Diagnostic species; Thymus longicaulis subsp. chaubardii (Rchb.f.) Jalas [Syn: Thymus longicaulis C. Presl subsp. subisophyllus (Borbás) Jalas], Alyssum virgatum Nyár., Astragalus leucotrix Freyn. \& Bornm., Helichrysum arenarium L. Moench. subsp. aucheri (Boiss.) Davis \& Kupicha, Erysimum thyrsoideum Boiss., Paronychia beauverdii Czecz., Silene olympica Boiss., Astragalus plumosus DC. ex Bunge subsp. nitens (Freyn \& Bornm.) Ponert

2.3.2. Order: ASPERULO PHRYGIAE-THYMENETALIA CHAUBARDII Akman, Quézel, Barbéro, Ketenoğlu \& Aydoğdu 1991

This suborder extends supramediterranean/temperate zone/Mediterranean orobiome between 1300 and $2000 \mathrm{~m}$ on siliceous and partly calcareous bedrock in south-west of Anatolia.

Characteristic \& Diagnostic species; Acantholimon acerosum (Willd.) Boiss. subsp. brachystachyum (Boiss.) Doğan \& Akaydın, Astragalus tmoleus Boiss. var. bounacanthus (Boiss.) D.F.Chamb., Acantholimon ulicinum (Willd. ex Schult.) Boiss. subsp. ulicinum, Asyneuma compactum Damboldt, Achillea wilhelmsii K.Koch, Cyanus pichleri (Boiss.) Holub. [Syn: Centaurea pichleri subsp. pichleri], Eremogone acerosa (Boiss.) Ikonn. [Syn: Arenaria acerosa Boiss. \& Heldr.], Cerasus prostrata (Labill.) Ser. var. prostrata (Moris) Browicz, Asperula lilaciflora subsp. Phrygia (Bornm.) Schönb.-Tem., Dianthus zonatus Fenzl. subsp. zonatus, Euphorbia kotschyana Fenzl., Erysimum smyrnaeum Boiss. \& Balansa, Paronychia dudleyi Chaudhri, Festuca paphlagonica (St.-Yves) Markgr.Dann. subsp. paphlagonica, Thymus longicaulis C.Presl subsp. chaubardii (Rchb.f.) Jalas Hypericum origanifolium Willd., Saponaria prostrata Willd. subsp. prostrata, Plantago holosteum Scop.

2.3.2.1. Alliance: Siderito phrygiae-Centaurion maculicipis Akman, Quézel, Barbéro, Ketenoğlu \& Aydoğdu 1991

Locality; Sultan Mountains/Akşehir (Akman et al, 1991)

Bedrock; Phyllite, calcareous

Altitude; $1200-2200 \mathrm{~m}$

Characteristic \& Diagnostic species; Asperula nitida subsp. hirtella, Astragalus strictispinis Boiss., A. wiedemannianus Fischer, Aubrieta anamasica, Bolanthus frankenioides var. fasciculatus, Centaurea cariensis Boiss. var. maculiceps (O.Schwarz) Wagenitz, Helictotrichon pubescens subsp. longipes, Minuartia anatolica (Boiss.) Woron. subsp. anatolica, Minuartia leucocephala (Boiss.) Mattf., Sideritis phrygia Bornm., Verbascum cherianthifolium Boiss. subsp. heldreichii.

2.3.2.2. Alliance: Verbasco phrygiae-Astragalion flavescentis Akman, Quézel, Barbéro, Ketenoğlu\&Aydoğdu 1991

Locality; Kumalar Mountain/Afyon (Akman et al, 1991)

Bedrock; Desite, Andesite

Altitude; $1200-2300 \mathrm{~m}$

Characteristic \& Diagnostic species; Astragalus flavescens Boiss., Euphorbia anacampseros Boiss. subsp. anacampseros, Sideritis condensata Boiss. \& Heldr. Apud Bentham, Verbascum cryserium Schrad., Verbascum phryginum Bornm.

2.3.2.3. Alliance: Astragalo akscheriensis-Onobrychidion pisidici Akman, Quézel, Barbéro, Ketenoğlu\&Aydoğdu 1991

Locality; Karadağ/Isparta (Akman et al, 1991)

Bedrock; Volcanic

Altitude; $1300-2300 \mathrm{~m}$

Characteristic \& Diagnostic species; Astragalus akscheherensis Freyn. \& Bornm., Chamaecytisus drepanolobus (Boiss.) Rothm., Hieracium patentissimum Freyn \& Sint. ex Freyn \& Sint., Onobrychis pisidica Boiss., Sideritis pisidica Boiss. \& Heldr. Apud Bentham, Saponaria chlorifolia (Poir.) Kunze

2.3.2.4. Alliance: Micromerio phrygiae-Olympociadion caespitosi Akman, Quézel, Barbéro, Ketenoğlu \& Aydoğdu 1991

Locality; Kap1 Mountain, Barla Mountain/Isparta (Akman et al, 1991)

Bedrock; Calcareous

Altitude; $1950-2250 \mathrm{~m}$ 
Characteristic \& Diagnostic species; Cyclothrium origanifolium (Labill.) Manden. \& Scheng., Festuca jeanpertii (St. Yves) F. Markgraff Apud Hayek subsp. jeanpertii, Micromeria cristata (Hampe) Griseb. subsp. phrygia P.H. Davis, Olymposciadium caespitosum (SM.) Wolff., Silene caryophylloides (Poiret) Otth. subsp. eglandulosa (Chowdh.) Coode \& Cullen

2.3.2.5. Alliance: Genisto involucratae-Marrubion micranthi Akman, Vural, Quézel, Kurt, Ketenoğlu, Serin \& Barbéro 1996

Locality; Karaman-Ermenek (Akman et al, 1996)

Bedrock; Calcareous, calcareous marl

Altitude; $1450-1650 \mathrm{~m}$

Characteristic \& Diagnostic species; Marrubium globosum subsp. micranthum Montbret \& Aucher ex Benth., Genista involucrata Spach., Cousinia ermenekensis Hub.-Mor., Arenaria ledebouriana var. parviflora Boiss., Astragalus acicularis (Bunge) Podl., Paronychia argyroloba Stapf.

2.4. Order: HYPERICO-THYMETALIA SKORPILII Akman, Quézel, Yurdakulol, Ketenoğlu \& Demirörs 1987

This order spreads in the western part of Ilgaz Mountains, encloses the highest peaks of the Bolu region (Abant, Aladağ and Çaltepe) in the northwest of the Anatolia and also is seen on Kısır Mountains (Kars) in the northeastern part of Anatolia.

Characteristic \& Diagnostic species; Thymus praecox subsp. skorpilii (Velen.) Jalas, Hypericum linarioides Bosse, Veronica gentianoides Vahl, Campanula olympica Boiss., Potentilla crantzii (Crantz) Beck ex Fritsch. var. crantzii, Bupleurum falcatum subsp. persicum (Boiss.) H.Wolff, Pilosella hoppeana subsp. troica (Zahn) P.D.Sell \& C.West, Bunium microcarpum subsp. bourgaei (Boiss.) Hedge \& Lamond, Scabiosa columbaria L. subsp. pahlagonica, Asperula capitellata Hausskn. \& Bornm., Allium olympicum Boiss., Dianthus balansae Boiss, Anthemis melanoma Trautv. subsp. melanoma, Sesleria pontica Deyl, Pilosella macrotricha (Boiss.) F.W.Schultz \& Sch.Bip., Stachys germanica subsp. bithynica (Boiss.) R.Bhattacharjee, Scorzonera pygmaea subsp. nutans (Czeczott) D. F.Chamb.

2.4.1. Alliance: Sileno-Astragalion densifolii Akman, Quézel, Yurdakulol, Ketenoğlu \& Demirörs 1987

Locality; Northern slopes of Ilgaz Mountains/Kastamonu, Akyaka, Arpaçay, Melikköy and Değirmenköprüköy Plateaus/Kars (Akman et al, 1983; Ocakverdi, 1994)

Bedrock; Siliceous

Altitude; $1850-2200 \mathrm{~m}$

Characteristic \& Diagnostic species; Silene olympica Boiss., Erysimum pulchellum (Willd.) J.Gay, Astragalus densifolius Lam., Stachys byzantina K.Koch, Onobrychis montana subsp. cadmea (Boiss.) P.W.Ball, Muscari aucheri (Boiss.) Baker, Anthemis tinctoria subsp. pallida DC., Minuartia hirsuta subsp. falcata (Griseb.) Mattf., Ranunculus dissectus M. Bieb. subsp. sibthorpii Davis, Nepeta nuda subsp. albiflora (Boiss.) Gams, Achillea coarctata Poir.

2.4.1.1. Suballiance: Globulario cordifoliae-Dianthenion leucophae Akman, Quézel, Barbéro, Aydoğdu, Demirörs \& Ekim 1988

Locality; Keltepe/Karabük (Akman et al, 1988)

Bedrock; Calcareous

Altitude; 1600-2000 m

Characteristic \& Diagnostic species; Globularia cordifolia L., Alchemilla sericata Rchb. ex Buser, Sideritis dichotoma Huter, Dianthus leucophaeus Sm., Valeriana phu L.

2.4.2. Alliance: Peducularo-Asterion alpinii Akman, Quézel, Yurdakulol, Ketenoğlu and Demirors 1987

Locality; Ilgaz Mountains, Akyaka, Arpaçay, Melikköy and Değirmenköprüköy Plateaus, Kısır Mountains/Kars (Ocakverdi 1994; Ocakverdi et al, 2009)

Bedrock; Calcareous, volcanic

Altitude; 2500-3000 m 
Characteristic \& Diagnostic species; Pedicularis comosa L. subsp. sibthorpii (Boiss.) Boiss., Gentiana verna subsp. balcanica N.M. Pritch., Aster alpinus L., Myosotis alpestris F.W. Schmidt, Jasione supina subsp. pontica (Boiss.) Damboldt, Gentiana septemfida Pall., Galium album Mill. subsp. prusense (K.Koch) Ehrend.\&Krendl.

\subsection{Order: FESTUCO OREOPHILAE-VERONICETALIA ORIENTALIS Hamzaoğlu 2006}

This order extends to the mountains in the west of Anatolian Diagonal, Eastern Pontic Mountains and southeast Taurus Mountains. The associations included in the order are found between 1500 and $3200 \mathrm{~m}$. The order spreads on volcanic bedrocks limestone and marl sediments.

Characteristic \& Diagnostic species; Arenaria dianthoides Sm., Astragalus lagurus Willd., Astragalus onobrychis L., Coronilla orientalis Mill. var. orientalis, Festuca brunnescens (Tzvelev) Galushko, Medicago papillosa Boiss., Onobrychis transcaucasica Grossh., Rosa pimpinellifolia L., Scutellaria orientalis L. subsp. orientalis, Thymus transcaucasicus Ronniger, Veronica orientalis Mill. subsp. orientalis, Acantholimon caryophyllaceum Boiss. subsp. caryophyllaceum, Alyssum pateri Nyár. subsp. prostratum (Nyár.) T.R. Dudley, Anemone albana Steven subsp. armena (Boiss.) Smirn., Artemisia spicigera K.Koch, Asperula laxiflora Boiss., Astragalus cinereus Willd., Centaurea rhizantha C.A.Mey, Coluteocarpus vesicaria (L.) Holmboe subsp. vesicaria, Cephalaria sparsipilosa V.A.Matthews, Daphne oleoides Schreb. subsp. kurdica (Bornm.) Bornm., Erysimum leptocarpum Gay, Festuca oreophila Markgr.- Dann., Gypsophila bitlisensis W.F.Barker, Helichrysum arenarium (L.) Moench subsp. rubicundum (K.Koch) P.H.Davis \& Kupicha, Malabaila dasyantha (K.Koch) Grossh., Pimpinella peucedanifolia Fisch. ex Ledeb., Silene montbretiana Boiss., Thymus pubescens Boiss. \& Kotschy var. cratericola Jalas.

2.5.1 Alliance: Festuco oreophilae-Veronicion orientalis Hamzaoğlu 2006

Locality; Nemrut Mountains/Bitlis, Allahuekber Mountains/Kars-Erzurum, Dumlu Mountain /Erzurum, Ağrı and Tahir Mounatins/Ağrı, Hizan, Varik Plateau/Bitlis, Akyaka, Arpaçay, Melikköy and Değirmenköprüköy Plateaus/Kars (Tatlı 1987; Behçet \& Tatl1 1989; Gümüş 1992; Behçet 1994a; Ocakverdi 1994)

Bedrock; Volcanic, limestone

Altitude; $1650-2650 \mathrm{~m}$

Characteristic and differential species are counted in the order Festuco oreophilae-Veronicetalia orientalis.

2.5.2 Alliance: Tanaceto aucherani-Thymion pubescentis Hamzaoğlu 2006

Locality; Çoruh and Kelkit Valleys/Gümüşhane-Bayburt (Hamzaoğlu, 2006)

Bedrock; Limestone, volcanic and marl

Altitude; $1500-2200 \mathrm{~m}$

Characteristic \& Diagnostic species; Achillea schischkinii Sosn., Astragalus lagopodioides Vahl, Centaurea carduiformis DC. subsp. orientalis Wagenitz, Isatis candolleana Boiss., Tanacetum aucheranum (DC.) Sch.Bip., Thymus pubescens Boiss. \& Kotschy var. pubescens, Arenaria armeniaca Boiss., Erysimum pycnophyllum Gay subsp. ponticum (Hausskn. \& Bornm.) Cullen, Onobrychis hajastana Grossh., Salvia rosifolia Sm., Senecio lorentii Hochst.

2.5.3. Alliance: Astragalo aurei-Festucion caucasicae Hamzaoğlu 2006

Locality; Dumlu, Gavur and Palandöken Mountains/Erzurum, Pirreşit Mountain/Van, Süphan and Nemrut Mountains/Bitlis (Behçet and Tatl1, 1989; Tatl1, 1985; Behçet and Ünal, 1999)

Bedrock; Andesite, basalt (volcanic)

Altitude; 2400-3200 m

Characteristic \& Diagnostic species; Astragalus aureus Willd., Cephalaria procera Fisch. \& AvéLall., Festuca woronowii Hack. subsp. caucasica (St.-Yves) Markgr.- Dann., Nepeta transcaucasica Grossh., Silene arguta Fenzl, Vicia alpestris Steven subsp. alpestris, Erigeron caucasicus Stev. subsp. venustus (Botsch.) Grierson, Poa longifolia Trin., Senecio pseudo-orientalis Schischk.

2.6. Order TRIFOLIO ANATOLICI-POLYGONETALIA ARENASTRI Quézel 1973

This order includes meadows in Taurus Mountains. 
Characteristic \& Diagnostic species; Crypsis acuminata Trin., Astragalus acmonotrichusFenzl, Agrostis stolonifera L., Astragalus tauricolus Boiss., Eremopoa persica (Trin.) Roshev. [Syn: Poa persica Trin.], Astragalus pinetorum Boiss., Polygonum cognatum Meisn., Plantago lanceolata L., Polygonum arenastrum Boreau, Plantago media L., Ranunculus demissus DC. var. major Boiss., Helichrysum plicatum DC., Paronychia polygonifolia (Vill.) DC., Pilosella macrotricha (Boiss.) F.W.Schultz \& Sch.Bip., Lotus corniculatus var. alpinus Ser., Taraxacum officinale (L.) Weber ex F.H.Wigg., Potentilla hirta L., Astragalus gymnolobus Fisch., Trifolium hybridum var. anatolicum (Boiss.) Hossain, Astragalus caudiclosus Boiss., Taraxacum bithynicum DC., Myosotis alpesteris F.W.Schmidt

2.6.1. Alliance Thlaspion papillosi Kürschner, Parolly \& Raab-Straube 1998

Locality; Bey Mountains, Akdağ, Bakırlı Mountain/Western Taurus, Geyik Mountains/West of Central Taurus (Kürschner et al, 1998)

Bedrock; Calcareous

Altitude; $2180-2720 \mathrm{~m}$

Characteristic \& Diagnostic species; Corydalis erdelii Zucc., Gagea villosa (M. Bieb.) Sweet var. hermonis Dafn \& Heyn, Marrubium bourgaei Boiss., Ornithogalum oligophyllum E.D. Clarke [Syn: Ornithogalum brevipedicellatum Boiss. ex Baker], Scilla bifolia L. [Syn: Scilla pleiophylla Speta], Noccaea papillosa (Boiss.) F.K. Mey. [Syn: Thlaspi papillosum Boiss.], Tragopogon olympicus Boiss., Veronica cuneifolia D.Don subsp. cuneifolia, Veronica cuneifolia subsp. isaurica P.H.Davis

2.6.2. Alliance: Bolanthion frankenioidis Quézel em. Kürschner, Parolly \& Raab-Straube 1998

Locality; Bey Mountains, Akdağ/Western Taurus Mountains (Kürschner et al, 1998)

Bedrock; Calcareous

Altitude; 1900-2700 m

Characteristic \& Diagnostic species; Bolanthus frankenioides (Boiss.) Barkoudah, Tanacetum densum (Labill.) Sch.Bip., Astragalus angustifolius Lam., Veronica cuneifolia D.Don, Spergularia lycia P.Monnier \& Quézel

2.6.3. Alliance: Trifolio-Polygonion Quézel 1973

Locality; Akseki, Oyuklu Mountain, Bolkar Mountains, Acıman Plateau/Central and Eastern Taurus Mountains (Quézel, 1973)

Bedrock; Serpantine

Altitude; 2000-2400 m

Characteristic \& Diagnostic species; Helichrysum plicatum DC., Lotus corniculatus var. alpinus Ser., Polygonum arenastrum Boreau, Marrubium lutescens Boiss. \& Heldr. micranthum (Boiss. \& Heldr.) P.H. Davis [Syn: Marrubium micranthum Boiss. \& Heldr.], Trifolium hybridum subsp. anatolicum (Boiss.) Hossain, Polygonum cognatum Meisn., Astragalus gymnolobus Fisch., Ranunculus demissus DC. var. major Boiss., Taraxacum officinale (L.) Weber ex F.H.Wigg., Eremopoa persica (Trin.) Roshev. [Syn: Poa persica Trin.], Potentilla hirta L., Paronychia polygonifolia (Vill.) DC., Astragalus angustifolius Lam., Astragalus acmonotrichus Fenzl

3. Class: ALCHEMILLO-CAMPANULETEA TRIDENTATA (Quezel \& Düzenli 1979)

3.1. Order: ALCHEMILLO-CAMPANULETALIA TRIDENTATAE (Quezel \& Düzenli 1979)

This order includes alpine meadows that have Euxin and Hyrcano-Euxin elements of Northeastern Anatolia and Caucasus and it is also present in two types of environment: wet, substantially fixed stony ground and the dry, surface (shallow), very mobile and stony ground (Düzenli, 1988; Korotkov and Belonovskaya, 2004).

Locality; Tiryal Mountain, Artvin

Bedrock; Spilite, dacite

Altitude; 2100-2844 m

Characteristic \& Diagnostic species; Alchemilla caucasica Buser, Minuartia recurva (All.) Schinz \& Thell. subsp. oreina (Mattf.) McNeill, Campanula tridentata Schreb., Carum caucasicum (M.Bieb.) Boiss., Ranunculus brachylobus Boiss. \& Hohen. subsp. brachylobus, Poa alpina L. [Syn: 
Poa alpina subsp. fallax F.Hermann], Thymus nummularius M.Bieb., Primula algida Adams, Erigeron caucasicus Steven subsp. venustus (Botsch.) Grierson, Carex cilicica Boiss., Trifolium polyphyllum (C.A.Mey.) Latsch., Tripleurospermum oreades (Boiss.) Rech.f., Pedicularis pontica Boiss., Viola altaica Ker Gawl. subsp. oreades (M. Bieb.) W.Becker, Heracleum apifolium Boiss.

3.1.1. Alliance: Stachydion macranthae (Quezel\&Düzenli 1979)

Locality; Tiryal Mountain/Artvin (Düzenli 1976)

Bedrock; Flysch

Altitude; 2100-2844 m

Characteristic \& Diagnostic species; Stachys macrantha (K.Koch) Stearn, Polygonum bistorta L., Geranium subacutum (Boiss.) Aedo [Syn: Geranium cinereum subsp. subcalescens (Boiss.) P.H.Davis \& J.Roberts], Anthyllis vulneraria subsp. boissieri (Sagorski) Bornm., Astragalus viridissimus Freyn \& Sint., Pedicularis pontica Boiss., Trifolium ambiguum M.Bieb., Draba hispida Willd.

3.1.2. Alliance: Oxytropion albanae (Quezel\&Düzenli 1979)

Locality; Tiryal Mountain/Artvin (Düzenli, 1976)

Bedrock; Flysch

Altitude; 2100-2844 m

Characteristic \& Diagnostic species; Oxytropis albana Steven, Cerastium gnaphalodes Fenzl, Androsace villosa L., Anthemis marschalliana subsp. pectinata (Boiss.) Grierson

4. Class: ASPLENIETEA TRICHOMANIS (Br.-Bl. in Meier and Br.-Bl. 1934) Oberd. 1977 This class as known chasmophytic vegetation on calcareous bedrocks is rich in endemic species and spreads on Taurus Mountains.

Characteristic \& Diagnostic species; Asplenium trichomanes L., Ceterach officinarum Willd., Asplenium ruta-muraria L., Cheilanthes pteridioides C. Chr. [Syn: Cheilanthes fragrans (L. f.) Sw.], Cystopteris fragilis (L.) Bernh., Parietaria judaica L., Arabis caucasica Willd. [Syn: Arabis alpina subsp. caucasica (Willd.) Briq.]

4.0.1. Subclass: POTENTILLENEA SPECIOSAE Hein, Kürschner \& Parolly 1998

Rock surfaces and crevices mountain ranges in Anatolia covered by chasmophytic plant communities are included in this subclass.

\subsection{SILENETALIA ODONTHOPETALEA Quézel 1973}

This order mostly extends on calcareous slopes of high mountains in Antalya province and environs and also central and eastern Taurus Mountains. The order comprises five alliances that are in the xero-to mesophytic montane to subalpine and hygro-to mesophytic oreal to alpine zone in the South Anatolia and one alliance in Northwest Anatolia (Hein et al. 1998). Characteristic species determined so far of the order is not enough, thus, exhaustive studies in the future will be allowed to increase the number of species belonging to alliances and associations (Akman et al, 2014).

Characteristic \& Diagnostic species; Asplenium haussknechtii Godet \& Reut., Rosularia sempervivum (M.Bieb.) A. Berger subsp. pestalozzae (Boiss.) Eggli [Syn: Rosularia pestalozzae (Boiss.) Samuelsson \& Froderstrom], Arenaria tmolea Boiss., Potentilla kotschyana Fenzl, Arenaria deflexa Decne., Salvia caespitosa Montbret \& Aucher ex Benth., Silene odontopetala Fenzl, Macrotomia densiflora (Ledeb. ex Nordm.) J.F.Macbr. [Syn: Macrotomia cephalotes Boiss.], Dianthus elegans d'Urv. subsp. actinopetalus (Fenzl) Reeve, Hypericum origanifolium Willd., Campanula cymbalaria Sm., Ranunculus libanoticus Freyn, Tanacetum nivale Sch.Bip., Asyneuma compactum Damboldt, Hieracium pannosum Boiss.

4.1.1. Alliance: Aubretion olympicae Quézel \& Pamukçuoğlu 1970

Locality; Uludağ and environs

Characteristic \& Diagnostic species; Aubrieta olympica Boiss., Scrophularia olympica Boiss, Lamium garganicum subsp. striatum (Sm.) Hayek, Saxifraga rotundifolia L.

4.1.2. Alliance: Silenion odontopetalae Quézel 1973

Locality; Bakırlı Mountain/Western Taurus Mountains, Çiçek and Davras Mountains/Isparta (Quézel, 1973; Sağlam, 2007; Sağlam, 2014) 
Bedrock; Calcareous

Altitude; 1940-2500 m

Characteristic \& Diagnostic species; Silene odontopetala Fenzl, Asyneuma linifolium (Boiss. \& Heldr.) Bornm. subsp. linifolium, Laserpitium petrophilum Boiss. \& Heldr., Hypericum origanifolium var. depilatum (Freyn \& Bornm.) N.Robson [Syn: Hypericum avicularifolium Jaub. \& Spach. subsp. depilatum Freyn \& Bornm.]

4.1.3. Alliance: Onosmion mutabilis Quézel 1973

Locality; Bolkar Mountains and Aladağlar/East of Central Taurus Mountains, Engizek Mountain/Kahramanmaraş (Quézel, 1973; Duman, 1995)

Bedrock; Calcareous

Altitude; $1500-2700 \mathrm{~m}$

Characteristic \& Diagnostic species; Arabis aubrietioides Boiss., Scrophularia variegata M.Bieb., Aethionema capitatum Boiss. \& Balansa, Veronica macrostachya Vahl subsp. sorgerae M.A.Fisch., Potentilla speciosa Willd., Tanacetum argenteum subsp. flabellifolium (Boiss. \& Heldr.) Grierson, Tanacetum armenum (DC.) Sch.Bip., Onosma mutabile Boiss. \& Hausskn.

4.1.4. Alliance: Campanulion isauricae Hein, Kürschner and Parolly 1998 1998)

Locality; Geyik Mountains, Oyuklu Mountain/Western Central Taurus Mountains (Hein et al,

Bedrock; Limestone

Altitude; $1850-2550 \mathrm{~m}$

Characteristic \& Diagnostic species; Arenaria tmolea Boiss., Hypericum origanifolium Willd., Prometheum chrysanthum (Boiss.) 't Hart subsp. chrysanthum, Valeriana speluncaria Boiss., Nepeta concolor Boiss. \& Heldr. ex Benth., Onosma isaurica Boiss. \& Heldr., Asyneuma compactum Damboldt, Campanula isaurica Contandr., Quézel \& Pamukç., Campanula leucosiphon Boiss. \& Heldr., Erodium pelargoniflorum Boiss. \& Heldr., Euphorbia isaurica M.L.S.Khan, Geranium glaberrimum Boiss. \& Heldr., Senecio castagneanus DC.

4.1.5. Alliance: Drabion acaulis Hein, Kürschner and Parolly 1998

Locality; Bolkar Mountains/Eastern Central Taurus Mountains (Hein et al, 1998)

Bedrock; Limestone

Altitude; 2600-3720 m

Characteristic \& Diagnostic species; Potentilla pulvinaris Fenzl, Draba acaulis Boiss., Veronica kotschyana Benth., Minuartia rimarum Mattf., Thymus leucotrichus Halácsy

4.1.6. Alliance: Campanulion cymbalariae Hein, Kürschner and Parolly 1998

Locality; Bakır Mountains, Elmalı Village/ Western Central Taurus Mountains, Aladağlar, Bolkar Mountains/Eastern Central Taurus Mountains (Hein et al, 1998)

Bedrock; Limestone

Altitude; 2000-2900 m

Characteristic \& Diagnostic species; Campanula cymbalaria Sm., Saxifraga luteoviridis Schott $\&$ Kotschy, Pedicularis cadmea Boiss., Myosotis speluncicola (Boiss.) Rouy, Ranunculus demissus DC. var. major Boiss., Cystopteris fragilis (L.) Bernh., Arenaria deflexa Decne., Sedum magellense Ten., Umbilicus luteus (Huds.) Webb \& Berthel., Scrophularia cryptophila Boiss., Asplenium ramosum L., Alchemilla ellenbergiana Rothm., Oxyria digyna (L.) Hill , Poa cenisia All., Poa bulbosa L., Lactuca muralis (L.) Fresen., Anthriscus kotschyi Fenzl ex Boiss.

5. Class: HELDREICHIETEA Quézel ex Parolly 1995

This class includes plant communities on calcareous and serpentine moving rocks mountain ranges in South Anatolia (Quezel, 1973; Parolly, 1995). Besides, Parolly (1998) is stated that Düzenli (1976) identified two plant communities included in Heldreichietea in Hasandağ/Central Anatolia and there is a high possibility of occurence of scree communties within Heldreichietea in North Anatolian Mountain ranges. 
Characteristic \& Diagnostic species; Ajuga chamaepitys (L.) Schreb. subsp. glareosa P.H.Davis, Alyssum argyrophyllum Schott \& Kotschy, Androsace villosa L., Arabis alpina subsp. brevifolia (DC.) Greuter \& Burdet, Bunium microcarpum (Boiss.) Freyn \& Bornm., Bupleurum falcatum L., Cicer incisum (Willd.) K.Maly, Euphorbia herniariifolia Willd. var. glaberrima Halácsy, Galium cilicicum Boiss., Galium incanum Sm., Heldreichia subsp., Lamium garganicum subsp. striatum (Sm.) Hayek [Syn: Lamium garganicum subsp. reniforme (Montbret \& Aucher ex Benth.) R.R.Mill], Laserpitium petrophilum Boiss. \& Heldr., Oxyria digyna (L.) Hill, Ranunculus brevifolius Ten., Rumex scutatus L., Noccaea sintenisii (Bornm.) F.K. Mey. [Syn: Thlaspi sintenisii Bornm.], Vavilovia formosa (Steven) Al.Fed., Veronica caespitosa Boiss. var. caespitosa, Viola crassifolia Fenzl

5.1. Order: LAMIETALIA CYMBALARIIFOLII Parolly 1995

This order includes plant communities on moving rocks in the western Taurus.

Characteristic \& Diagnostic species; Lamium garganicum var. microphyllum (Boiss.) Mennema [Syn: Lamium cymbalariifolium Boiss.], Scrophularia depauperata Boiss., Fritillaria crassifolia Boiss. \& A.Huet subsp. crassifolia, Ranunculus cadmicus Boiss., Aethionema cordatum (Desf.) Boiss., Euphorbia pestalozzae Boiss., Festuca adanensis Markgr.-Dann., Galium incanum subsp. centrale Ehrend., Heldreichia bupleurifolia Boiss. subsp. bourgaei (Boiss.) Parolly, Nordt \& Mumm., Ricotia davisiana B.L. Burtt, Scrophularia candelabrum Heywood, Vavilovia formosa (Steven) Al.Fed., Euphorbia herniariifolia Willd. var. glaberrima Halácsy, Peucedanum alpinum (Sieber ex Schult.) B.L.Burtt \& P.H.Davis

5.1.1. Suborder: LAMIENALIA CYMBALARIIFOLII Parolly 1995

5.1.1.1. Alliance: Scrophularion depauperetae Parolly 1995

Locality; Akdağ, Bey Mountains/Western Taurus Mountains (Parolly, 1995)

Bedrock; Limestone

Altitude; 2000-3000 m

Characteristic \& Diagnostic species; Alkanna attilae P.H.Davis, Heldreichia bupleurifolia Boiss. subsp. bourgaei (Boiss.) Parolly, Nordt \& Mumm, Scrophularia depauperata Boiss., Cyanus bourgaei (Boiss.) Wagenitz \& Greuter, Elymus lazicus (Boiss.) Melderis, Lamium garganicum var. microphyllum (Boiss.) Mennema [Syn: Lamium cymbalariifolium Boiss.], Euphorbia pestalozzae Boiss., Ormosolenia alpina (Sieber ex Schult.) Pimenov

5.2. Order: HELDREICHIETALIA Quézel ex Parolly 1995

This order includes plant communities on moving rocks on mountain ranges in the central and southern Taurus.

Characteristic \& Diagnostic species; Cerastium cerastoides (L.) Britton, Cyclotrichium origanifolium (Labill.) Manden. \& Scheng., Galium incanum subsp. elatius (Boiss.) Ehrend., Heldreichia bupleurifolia Boiss. subsp. bourgaei (Boiss.) Parolly, Nordt \& Mumm, Heracleum humile Sm., Lamium eriocephalum Benth., Linaria corifolia Desf., Melica ciliata L., Nepeta cilicica Boiss. ex Benth., Poa cenisia All., Scorzonera cana (C.A.Mey.) Griseb.

5.2.1. Suborder: LAMIENALIA GLANDULOSIDENTIS Parolly 1995

This suborder extends west of Central Taurus.

5.2.1.1. Alliance: Scrophularion myriophyllae Parolly 1995

Locality; Bakırlı Mountain/Central Taurus Mountains (Parolly, 1995)

Bedrock; Limestone

Altitude; 2160-2300 m

Characteristic \& Diagnostic species; Scrophularia myriophylla Boiss. \& Heldr., Lamium eriocephalum Benth., Heldreichia bupleurifolia Boiss., Ricotia varians B.L. Burtt

5.2.2. Suborder: LAMIENALIA ERIOCEPHALI Parolly 1995

This order spreads on west of eastern Taurus Mountains.

5.2.2.1. Alliance: Scrophularion rimarium Parolly 1995

Locality; Bolkar Mountains and Aladağlar/The upper oreal to subalpine zone of east of Central Taurus Mountains (Parolly, 1995) 
Bedrock; Limestone

Altitude; 2200-2700 m

Characteristic \& Diagnostic species; Scrophularia rimarum Bornm., Anthriscus kotschyi Fenzl ex Boiss, Heracleum humile Sm., Aurinia rupestris subsp. cyclocarpa (Boiss.) Cullen \& T.R.Dudley, Arenaria kotschyana Fenzl subsp. kotschyana, Hypericum crenulatum Boiss., Aethionema stylosum DC., Aethionema speciosum Boiss. \& A.Huet

5.2.2.2. Alliance: Jurinellion moschus Parolly 1995

Locality; The upper alpine zone to subnival belt of the Eastern Central Taurus Mountains (Parolly, 1995)

Bedrock; Limestone

Altitude; 2750-3600 m

Characteristic \& Diagnostic species; Jurinella moschus (Habl.) Bobrov subsp. moschus, Silene pruinosa Boiss. [Syn: Silene supina subsp. pruinosa Chowdhuri], Hedysarum erythroleucum Schott \& Kotschy ex Boiss., Thymus leucotrichus Halácsy, Astragalus pelliger Fenzl, Noccaea sintenisii (Bornm.) F.K. Mey. [Syn: Thlaspi sintenisii Bornm. subsp. crassum], Minuartia rimarum Mattf., Crepis frigida (Boiss. \& Balansa) Babc., Scorzonera cana var. radicosa (C.A. Meyer) Hoffm., Scutellaria orientalis L. subsp. alpina (Boiss.) O.Schwarz var. glandulosissima O.Schwarz, Scutellaria orientalis L. subsp. pinnatifida J.R. Edm., Androsace multiscapa Duby, Tulipa humilis Herb.

6. Class: JUNCETEA MARITIMI Braun-Blanq. in Braun-Blanq., Roussine and Nègre 1952

This class involves plant communities dominated by Gramineae on salt marhes. It has a wide distribution and spreads on the whole Mediterranean and Black Sea region in Anatolia.

Characteristic \& Diagnostic species; Juncus maritimus Lam., Juncus acutus L., Polypogon maritimus Willd. subsp. maritimus, Limonium angustifolium (Tausch) Turrill, Aeluropus littoralis (Gouan) Parl., Plantago maritima L., Tetragonolobus maritimus (L.) Roth, Carex extensa Gooden.

6.1. Order: JUNCETALIA MARITIMI Braun-Blanq. ex Horvatić 1934

The order spreads across Mediterranean region and Black Sea region in Turkey and called the halophytic grasslands. The order has less salty and carbonate-rich soils and also due to exposing to floods in rainy seasons, the soils remain moist in the summers (Hamzaoğlu and Aksoy 2009).

Characteristic \& Diagnostic species; ; Juncus maritimus Lam, Triglochin maritima L., Lotus corniculatus var. tenuifolius L., Scorzonera parviflora Jacq., Juncus gerardii Loisel. subsp. gerardii, Orchis palustris Jacq., Taraxacum bessarabicum (Hornem.) Hand.-Mazz.

6.1.1. Alliance: Juncion maritimi Braun-Blanq. ex Horvatić 1934

Locality; Cihanbeyli, Kırkışla, Bulak Lake/Konya, Erçek, Turna, Bostaniçi/Van (Yurdakulol and Ercoşkun, 1990; Behçet, 1994b)

Altitude; $1890 \mathrm{~m}$

Characteristic \& Diagnostic species; Juncus maritimus Lam, Halimione portulacoides (L.) Aellen, Lotus corniculatus var. tenuifolius L., Polypogon maritimus Willd. subsp. maritimus, Puccinellia distans (Jacq.) Parl. subsp. distans,

Cirsieto-Juncetum maritime Yurdakulol and Ercoskun 1990. Cihanbeyli, Kirkisla, Bulak Lake, Aksaray

6.1.2. Alliance: Inulo aucheranae-Elymion salsi Aydoğdu, Hamzaoğlu\&Kurt 2002

Locality; Salt Lake/ Aksaray, Seyfe Lake/Konya, around Ufaktepe drainage channel/Yeşilhisar and Sultansazlığı Marsh/Kayseri (Aydoğdu et al, 2002; Hamzaoğlu and Aksoy 2009)

Bedrock; Salty soils

Altitude; 930-1076 m

Characteristic \& Diagnostic species; Inula aucherana DC., Verbascum pyroliforme (Boiss. and Heldr.) O. Kuntze, Gladiolus halophilus Boiss. and Heldr., Allium sieheanum [Hausskn. ex] Kollmann, Scorzonera hieraciifolia Hayek, Hypericum salsugineum Robson and Hub.-Mor., Gypsophila oblanceolata Bark., Onosma halophilum Boiss. and Heldr., Salvia halophila Hedge., Elymus elongatus (Host) Runemark subsp. salsus Melderis 
7. Class: MOLINIO-ARRHENATHERETEA Tüxen 1937

This class includes nutrient-rich hygrophilous meadow communities in the western Taurus, Hizan/Bitlis, Pirreşit Mountain/Van, Allahüekber Mountains/Erzurum.

Characteristic \& Diagnostic species; Lotus corniculatus var. tenuifolius L., Plantago lanceolata L., Dactylis glomerata subsp. hispanica (Roth) Nyman, Polygonum cognatum Meisn., Lotus corniculatus L. subsp. corniculatus, Rumex acetosella L., Achillea millefolium L. subsp. millefolium, Trifolium repens L. subsp. repens, Lathyrus pratensis L., Trifolium pratense L. subsp. pratense, uncus persicus subsp. libanoticus (J.Thiébaut) Novikov \& Snogerup [Syn: Juncus gerardii subsp. libanoticus (J.Thiébaut) Snogerup]

7.1. Order: ARRHENATHERETALIA ELATIORIS Pawl. 1928

The order is present at Dumlu Mountains/Erzurum, between Akyaka, Arpaçay, Melikköy and Değirmenköprüköy Plateaus/Kars

Characteristic \& Diagnostic species; Festuca pratensis Huds., Phleum pratense L., Agrostis stolonifera L., Plantago major L.

7.1.1. Alliance: Molinio-Holoschoenion Br. - Bl. 1947

Locality; Pirreşit Mountain, Muradiye/Van

Bedrock; Andesite

Altitude; 2400-2500 m

Characteristic \& Diagnostic species; Molinia caerulea (L.) Moench, Pulicaria dysenterica (L.) Bernh. subsp. dysenterica, Tetragonolobus maritimus (L.) Roth, Carex panicea L., Eupatorium cannabinum L., Hypericum tetrapterum Fr. var. tetrapterum, Prunella vulgaris L.

\section{References}

Akman, Y. 1974. Evolution régressive de la végétation a étage du Pinus nigra ssp. pallasiana dans l'Anatolie Centrale dans un climat méditerranéen semi aride très froid. [Regressive evolution of the vegetation stage of Pinus nigra subsp. pallasiana in Central Anatolia under the influences of semi-arid very cold Mediterranean climate] Communications De la Faculté Des Sciences De L’université d'Ankara, Serie C (18): 1-6.

Akman, Y. and Ketenoğlu, O. 1976. The phytosociological and phytoecological investigation on the Ayaş mountains. Communications De la Faculté Des Sciences De L’université d'Ankara, Série C (20), 1-43.

Akman, Y. and Ketenoğlu, O. 1978. Phytosociological investigations of Köroğlu Mountain. Communications de la Faculte des sciences de l'Universite d'Ankara. C (2/22): 1-24.

Akman, Y., Yurdakulol, E. and Demirörs, M. 1983. The Vegetation of Ilgaz Mountains. Ecologia Mediterranea, 9 (2): 137 165.

Akman, Y., Ketenoğlu, O., Quézel, P., and Demirörs, M. 1984. A syntaxonomic study of steppe vegetation in Central Anatolia. Phytocoenologia, 12 (4): 563-584.

Akman, Y., Ketenoğlu, O. and Quézel, P. 1985. A new syntaxon from Central Anatolia. Ecologia Mediterranea, 11(2-3): 111121.

Akman, Y., Quézel, P., Yurdakulol, E., Ketenoğlu, O. and Demirörs, M. 1987. La végétation des hauts sommets de l'IIgaz Dağ. Ecologia Mediterranea, 13(1/2): 119-129.

Akman, Y., Quezel, P., Barbero, M., Aydoğdu, M., Demirors, M. and Ekim, T. 1988. La végétation du keltepe (région de Karabük). Ecologia Mediterranea, 14 (1-2): 149-154.

Akman, Y. 1990a. Etude de la végétation steppique des montagnes d'Aydos située au Nord-Ouest d'Ankara. Ecologia Mediterranea, 16: 223-230.

Akman, Y., Ekim, T., Büyükburç, U., Ketenoğlu, O. and Karagüllü, N. 1990b. Polatlı Acıkır alanında doğal meraların bitki sosyolojisi yönünden araştırılması. Tarla Bitkileri Merkezi Araştırma Enstitüsü Genel Yayın No: 1990/3, Araştırma Yayın No: 1990/2, Tarım Matbaas1, Ankara.

Akman, Y., Quézel, P., Barbéro, M., Ketenoğlu, O. and Aydoğdu, M. 1991. La végétation des steppes, pelouses écorchées et à xérophytes épineux de l'Antitaurus dans la partie sud-ouest de l'Anatolie. Phytocoenologia, 19: 391-428.

Akman, Y., Quézel, P., Aydoğdu, M., Ketenoğlu, O., Kurt, L. and Evren, H. 1994. A phytosociological research on the steppe vegetation of the Yapraklı mountains (Çankırı, Turkey). Ecologia Mediterranea, 20 (3-4): 1-7. 


\section{E. Özdeniz}

Akman, Y., Vural, M., Quézel, P., Kurt, L., Ketenoğlu, O., Serin, M. and Barbero, M. 1996. Etude de la végétation steppique de la région de Karaman et d'Ermenek (sud de L'Anatolie Centrale). Ecologia Mediterranea, 22 (3-4): 1-7.

Akman Y., Ketenoğlu O., Kurt L. and Vural M. 2014. İç Anadolu step vejetasyonu. Palme Yayınları: 350, Ankara.

Aydoğdu, M., Ketenoğlu, O., and Hamzaoğlu, E. 1999. New syntaxa from Cappadocia (Kırşehir, Türkiye). Israel Journal of Plant Sciences, 47 (2): 123-129.

Aydoğdu, M., Hamzaoglu, E. and Kurt, L. 2002. New halophytic syntaxa from Central Anatolia (Turkey). Israel Journal of Plant Sciences, 50 (4): 313-323.

Aydoğdu, M., Kurt, L., Hamzaoğlu, E., Ketenoğlu, O. and Cansaran, A. 2004. Phytosociological studies on salty steppe communities of the Central Anatolia, Turkey. Israel Journal of Plant Sciences, 52: 71-79.

Barbero, M., Bonin, G. and Quézel, P. 1975. Les pelouses écorchées des montagnes Circum-Méditerranèennes. Aperçu bioclimatique et syngénétique, leurs rapports avec les forêts d'altitude. Phytocoenologia, 1: 427-459.

Behçet, L. and Tatl1, A. 1989. A fitosociological research on Dumlu mountains (Erzurum) vegetation. Turkish Journal of Botany, 13: 398-417.

Behçet, L. 1994a. Hizan (Bitlis) vejetasyonu. Turkish Journal of Botany 18: 289-303.

Behçet, L. 1994b. "Erçek, Turna ve Bostaniçi (Van) Göllerinin Vejetasyonu”, Turkish Journal of Botany, 18 (4): 305-312.

Behçet, L. and Ünal, M. 1999. Pirreşit Dağı (Muradiye-Van) vejetasyonu. Tatlı A (ed), 1st International Symposium on Protection of Natural Environment and Ehrami Karaçam, Proceeding book: 101-120, 23-25th September 1999, Kütahya.

Duman, H. 1995. Vegetation of Engizek Mountain (Kahramanmaraş). Turkish Journal of Botany, 19: 179-212.

Düzenli, A. 1976. A phytoecological and phytosociological research on Tiryal Hasan Dağ. Journal of Forestry Research, 22 (2): 7-53.

Düzenli, A. 1988. Nouveaux syntaxons phytosociologiques pour la végétation de Turquie. Ecologia Mediterranea, 14: 143148.

Gemici, Y. 1988. Vegetation of Akdağ (Afyon-Denizli) and its environs. Turkish Journal of Botany, 12 (1): 8-57.

Geven, F., Adıgüzel, N., and Vural, M. 2010. İç Anadolu'dan (Ereğli-Karaman) Onobrychido armeni-Thymetalia leucostomi Ordosu İçin Yeni Bir Alyans. Ekoloji, 19 (74): 89-101.

Gümüş, İ. 1992. Tahir Dağları ve Güzeldere Havzası (Ağrı) step vejetasyonunun bitki sosyolojisi yönünden araștırılması. Turkish Journal of Botany, 16: 153-175.

Güner A., Aslan, S., Ekim, T., Vural, M. and Babaç, M.T. (eds.). 2012. Türkiye Bitkileri Listesi (Damarlı Bitkileri) Nezahat Gökyiğit Botanik Bahçesi ve Flora Araştırmaları Derneği Yayını: 763, Istanbul.

Hamzaoğlu, E. and Duran, A. 2004. A Phytosociological research on the degraded forest vegetation of Dinek Mountain (Kırıkkale). Gazi University Journal of Science, 17 (4), 1-13.

Hamzaoğlu, E., Aydoğdu, M., Kurt, L. and Cansaran, A. 2004. New syntaxa from the west part of Central Anatolia. Pakistan Journal of Botany, 36 (2): 235-246.

Hamzaoğlu, E. 2006. Phytosociological studies on the steppe communities of East Anatolia. Ekoloji, 15(61): 29-55.

Hamzaoğlu, E., and Aksoy, A. 2009. Phytosociological studies on the halophytic communities of Central Anatolia. Ekoloji, 18 (71): 1-14.

Hein, P., Kürschner, H., and Parolly, G. 1998. Phytosociological studies on high mountain plant communities of the Taurus mountains (Turkey) 2. Rock communities. Phytocoenologia, 28: 465-563.

Kaya, Ö.F. and Ketenoğlu, O. 2010. A syntaxonomical and synecological research on the steppe vegetation of the Karacadağ mountain (Şanlıurfa/Diyarbakır). Ecologia Mediterranea, 36 (1): 45-62.

Ketenoğlu, O., Quézel, P., Akman, Y. and Aydoğdu, M. 1983. New syntaxa on the gypsaceous formations in the Central Anatolia. Ecologia Mediterranea, 9 (3-4): 211-221.

Ketenoğlu, O., Kurt, L., Akman, Y. and Serin, M. 1996. A New Alliance from Central Anatolia "Minuartion juniperinopestalozzae". Turkish Journal of Botany, 20: 457-464.

Ketenoğlu, O., Kurt, L., Akman, Y., Aydoğdu, M., and Hamzaoğlu, E. 2000. Syntaxonomic research on the gypsicole vegetation Cappadocia, Turkey. Israel Journal of Plant Sciences, 48 (2): 121-128. 
Ketenoğlu, O., Aydoğdu, M., Kurt, L., Hamzaoğlu, E., Tuğ, G. N., and Aslantürk, N. 2008. New Syntaxa from Steppe Vegetation in Cappadocia, Turkiye. Anadolu University Journal of Science and Technology, 9 (1):123-133.

Kılınç, M. 1985. İç Anadolu-Batı Karadeniz geçiş bölgesinde Devrez Çayı ile Kızılırmak nehri arasında kalan bölgenin vejetasyonu. Turkish Journal of Botany, 9 (2): 315-357.

Korotkov, K., and Belonovskaya, E. 2004. Syntaxonomic status of the Caucasian alpine meadows. Annali Di Botanica, 4: 139154.

Kurt, L., Kurt F., Evren H., Karakaya A. (1999). Steppic Vegetation of the Eldivan Mountain (Çankır1/Turkey). Fırat University Turkish Journal of Science and Technology, 11: 49-57.

Kurt, L. 2002. The steppe vegetation of Emirdağ (Afyon/Turkey). Anadolu University Journal of Science and Technology, 3 (2): $257-270$

Kurt, L., Tuğ, G.N. and Ketenoğlu, O. 2006. Synoptic view of the stepe vegetation of Central Anatolia (Turkey). Asian Journal of Plant Science, 5: 733-739.

Kürschner, H., Parolly, G. and Raab-Straube, E.V. 1998. Phytosociological studies on high mountain plant communities of the Taurus Mountains (Turkey) 3. Snow-patch and meltwater communities. Feddes Repertorium, 109 (7-8): 581-616.

Ocakverdi, H. and Çetik, R. 1982. Phytosociological and phytoecological investigation of Sultan mountains-Doğanhisar region (Konya). Selçuk University Journal of Faculty of Science, 2: 73-89.

Ocakverdi, H. 1994. Akyaka, Arpaçay, Melikköy ve Değirmenköprüköy Yaylaları (Kars) ile Sovyet sınırı arasında kalan bölgenin bitki sosyolojisi ve ekolojisi yönünden araştırılması. Turkish Journal of Botany 18: 245-265.

Ocakverdi, H., Vural, M. and Adıgüzel, N. 2009. Vegetation of Kısır Dağı (Kars-Ardahan/Turkey). BioDiCon, 2/2: 1-37.

Parolly, G. 1995. Die Steinschuttfluren (Heldreichietea) des Westlichen und Mittleren Taurus (Turkei). Pflanzensoziologische, floristische und kologische Untersuchungen. Diss Bot: 247, Berlin/Stuttgart.

Parolly, G. 1998. Phytosociological studies on high mountain plant communities of the South Anatolian Taurus mountains 1. Scree plant communities (Heldreichietea): A synopsis. Phytocoenologia, 28: 233-284.

Quézel, P. 1964. Végétation des hautes montagnes de la Grèce méridionale. Plant Ecology, 12 (5): 289-385.

Quézel, P. and Pamukçuoğlu, A. 1970. Végétation des hautes montagnes d'Anatolie nord occidentale. Israel Journal of Botany, 19: $348-400$.

Quézel, P. 1973. Contribution a l'etude phytocoenologique du masif du Taurus. Phytocoenologia, 1 (2): 131-222.

Quézel, P., Barbero, M. and Akman, Y. 1992. Typification de syntaxa décrits en région méditerranéenne orientale. [Typification of syntaxa described in the eastern Mediterranean area]. Ecologia Mediterranea, 28: 81-87.

Sağlam, C. 2007. The steppe and rock vegetation of Davras Mountain and surroundings (Isparta). Dumlupınar Üniversitesi Fen Bilimleri Enstitüsü Dergisi, 14: 11-25.

Sağlam, C. 2014. Phytosociological Features of Çiçek Mountain and environs (Isparta, Turkey). Ekoloji, 23 (92): 19-37.

Serin, M., Ketenoğlu, M. and Küçüködük, M. 1996. Hacıbaba Dağı'nın (Karaman) ormansal vejetasyonun fitososyolojik ve fitoekolojik yönden incelenmesi, S.Ü. Fen Edebiyat Fakültesi Fen Dergisi, 13: 179-194.

Şanda, M.A., Küçüködük, M. and Serin, M. 2000. The steppe vegetation of Hadim (Konya) - Ermenek - Bucakkışla (Karaman) S.Ü. Fen Edebiyat Fakültesi Fen Dergisi, 1: 21-35.

Takhtajan, A. 1986. Floristic region of the world. University of California Press: 544, Los Angeles.

Tatl1, A. 1982. Festucion chalcophaeae - Doğu Anadolu subalpin ve alpin bölge vejetasyonunun yeni bir alyansı. TÜBITTAK VII. Bilim Kongresi, Bildiriler kitab1: 703-726. İzmir.

Tatl1, A. 1985. Phytosociological investigation on the vegetation of Gavur Mountains. Turkish Journal of Botany, 9: 531-564.

Tatl1, A. 1987. A phytosociological investigation on the Allahuekber Mountains. Turkish Journal of Botany, 11: 169-193.

Tatl1, A. 1991. Phytosociological investigation of vegetation of wind-eroded areas of Eastern Anatolia. Ecologia Mediterranea, 17: 161-168.

Tatl1, A., Eyce, B. and Serin, M. 1994. The vegetation of Kızılören, Çal and Loras mountains (Konya). Turkish Journal of Botany, 18: 267-288.

Weber, H.E, Moravec, J. and Theurillat, J.P. 2000. International code of phytosociological nomenclature. 3rd ed. Journal of Vegetation Science, 11: 739-768. 


\section{E. Özdeniz}

Yurdakulol, E. 1973. Phytosociologique and ecological research on the vegetation of The Pos Forest (Adanadistr. Karsant1) of The Anti-Taurus Mountain. Communications De la Faculté Des Sciences De L'université d'Ankara,, Seri C, 2:1-50.

Yurdakulol, E. and Ercoşkun, T. 1990. Ecological and syntaxonomical studies on the vegetation of arid lands in central Anatolia. Turkish Journal of Botany, 14: 109-12 